\title{
Monitorising the Distelle Water in Battery Using IOT
}

\section{G. Yuvaraj, V. Anusha Lakshmi}

\begin{abstract}
Now-days the electronic devices play a major role in day-to-day life. Where as in case of electricity, people are using it for $24 b y 7$ as of there were of having household appliances are of electronic devices. So if there is any power loss in meantime of running any electronic devices it may leads to damage, so to predict they were of using the battery to work instant after power loss. As we know that there are different types of battery that runs with distilled water. So in this paper we would like to discuss about how to control the batteries voltage using IOT (Internet of Things). It was of having low cost and reduces the human resources and time-efficiency and cost the system used in it was of Voltmeter. It were of using the Raspberry pi for monitoring \& updating the values. While they were of using Arduino, cloud for transmitting the data.
\end{abstract}

Keywords--- Raspberry pi, Internet of Things, Voltameter, Could Computing.

\section{INTRODUCTION}

Now-a-days the technology changes continuously, as of now the people were of using the equipment's instantly to reduce the time and efficiency as we know that the electronic device were of changes the man life as well.one of the device is of Generator[1]. It were used in day-to-day life to run the electronic devices for long extension of electronic devices and not to waste of human resources.

As we all know that generators are electronic energy storing device and transmitting of energy. When there is a power loss in households then they were of undergoing the chemical reaction in it depending upon the composition of the materials that were and converts into another form of energy into electronic power and transmitted to the electronic devices. Depending upon the chemical reaction it may change in power rate.

Let us extend the above topic such that of generators while it were of using the distilled water for generation of electrons and transmission of power.

There will be of loss of water due to electron transformation, while the process undergoes in closed vacuum tube, the water in content in it were invisible, as it plays an major role in generators, we have to look through it every time.

It were heard to go through it And also of its very difficult to buy at any instant of time while in case of household they will not have more effect while in case of large companies or any co-operation that were of using electricity 24 by 7 may loss a lot[7][5]. Instead of checking every time of its completion. While it were of providing an average time of the battery completion, but it may change many time due to

G. Yuvaraj, Assistant Professor, Department of Computer Science and Engineering, School of Computing, Vel Tech Rangarajan Dr. Sagunthala R\&D Institute of Science and Technology, Avadi, Chennai, Tamil Nadu, India. (e-mail: gyuvarajdce@gmail.com)

V. Anusha Lakshmi, UG Student, Department of Computer Science and Engineering, School of Computing, Vel Tech Rangarajan Dr. Sagunthala R\&D Institute of Science and Technology, Avadi, Chennai, Tamil Nadu, India. (e-mail: vasupallianusha7@gmail.com)
Manuscript received September 16, 2019..

usage of power, so it's very difficult to identify its hydration time .

By seeing the above issues in our paper we would like to produce a low cost monitoring of battery's using IoT and Raspberry pi and SIM. The raspberry pi were of using an core controller and an design system to that of the IoT for accessing to the mobile to given an message depending upon the volume of the distilled water using voltmeter

As of better understanding they were divided into different modules. II. Relationship with IOT, III .Flow diagram, methodology and explanation of it .IV. Code and Result, V. Conclusion.

\section{RELATIONSHIP WITH IOT}

As we know that Internet Of Things(IoT) were used to connect all the things in an single device and also control by it through the internet or may be also of cloud, but according to today's technology

Cloud plays an major role. While the IoT is the internet access using the sensors for changing the physical reactions to an electrical sensors and transform the information[2].as we know that the cloud is the low cost and high efficiency usage. While it were of maintain an IP address for store the data at required conditions[5]. And transmits the data from the given conditions to it while the IOT were used in controlling water flow in tanks, home automata, monitoring home appliance, etc.

\section{METHODOLOGY}

In this we will be of seeing the construction and working of the monitoring the distilled water using IOT. In this there will be of having block diagram, working of it.

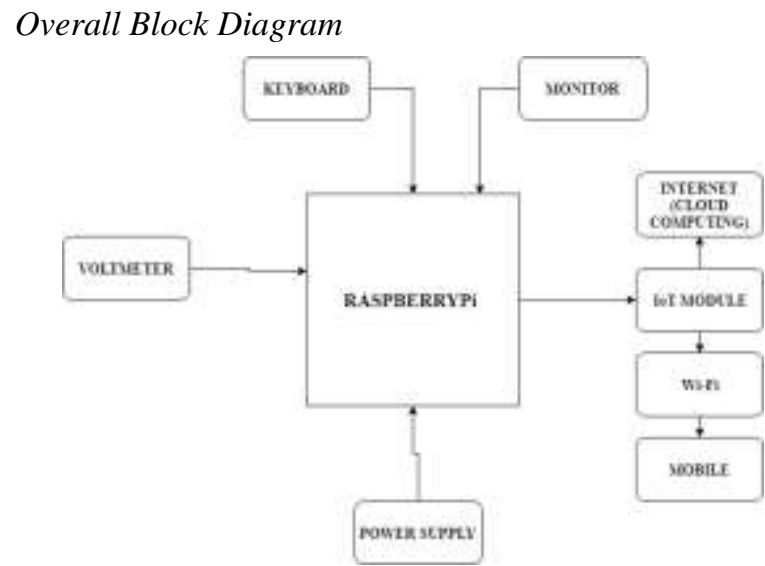

Fig. 3.1: overall block diagram for monitoring the Distell Water in Battery 


\section{MONITORISING THE DISTELLE WATER IN BATTERY USING IOT}

In the block diagram fig.1.1, it were of using the sensor like voltmeter were used to monitoring the change and send the information to the Raspberry pi, where as it used to controlling the values and transmit to the admin depend upon the condition given by the coder[3]. While there will be of using an Arduino to code the Raspberry pi and of also to store the values such that it were of used to create the data base.

\section{PROPOSED METHOD}

In our method Raspberry Pi were plays an major role in case controlling the input and output. It were of using LINUX kernel for monitoring IOT using the keyboard and mouse .It were of using the LINUX for boot of Raspberry into device and monitoring code through it to the board by giving command lines to it[3]. While in this case it was of using the code for Voltammeter to run through the device and show the changes in the distilled water level.

The Raspberry pi where of equipped with an range of driver through it. As we know that boot of every driver may not be possible at a time, so by increasing the boot time it may leads to the efficient result at last. Such that of installing all the modules[10]. Then they were of having an modules. They store the loadable modules and command and then data from Raspberry pi send the data to the IoT module

Then the data from the Internet Of Things(IoT) were sent through the internet by using data or by cloud that of used very vast range now-a-day and may be also sent through the WIFI connection for any mobile accessing device such that it were of monitoring,

The diagram is shown below

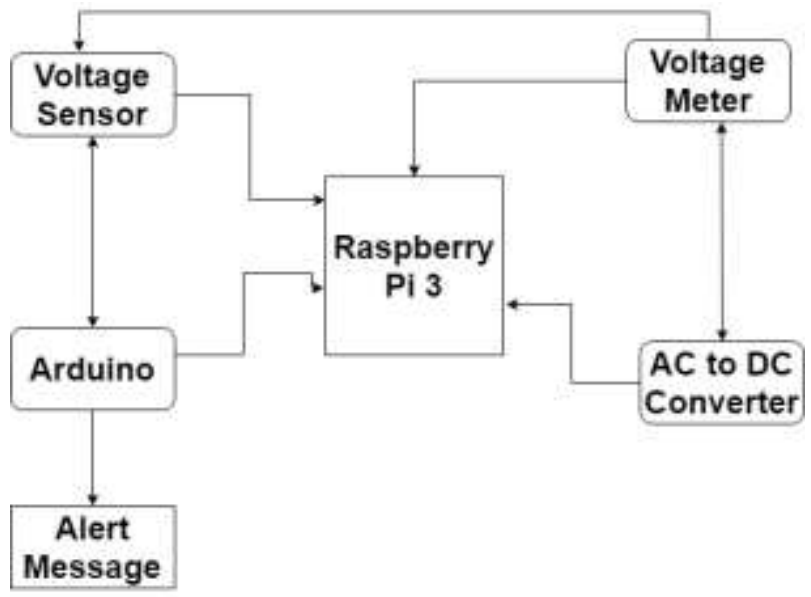

Fig. 4.1: collecting the voltage value and inform to admin

While after the they connect to the mobile then the IoT.

Then the information from sensor such as voltmeter were sent to the IoT and then recorded in it. While there will be of transmit the data through a gateway. While the Gateway is responsible for transmitting the data packets. These were of stored in the data base. While the voltage sensor is connected to the voltmeter that if any changes occurs in battery it will send and send information in the form of signals to voltmeter[11]. Then the values were sent to the Arduino. While the value in the database reaches to the limit that were given to the Raspberry Pi then the information mod probe after completion they were inserted into LINUX,

sent to the admin in the name of Alert Message as it was shown in the diagram.

\section{ALGORITHM \& RESULTS}

While there we were of using the Raspberry so it were of using the code for storing the values and convert the values from the voltmeter to the phone in an understanding way by the admin. As we of discussed above Raspberry of using many drives to compile the LINUX and store the values of it[9][8]. Where as it were coding the Raspberry pi using Arduino such let's see the steps or Algorithm used for coding and compiling the Raspberry pi in python while they were of using the mouse and keyboard.

The sequentially steps that are followed by it were of;

\section{Code for Drivers}

Step 1: Write the code; such that of installing the needed packages for the Rasp berry Pi.

\subsection{While they were of: \\ sudo apt-get update \\ sudo apt-get install python-dev python-setuptools}

sudo easy_install rpi.gpio

the above were used to get install of python for coding

step 1.2: Write the code for converting of analog input to digital converter that are understand by the system:

installing SPI such as: Adafruit_GPIO.SPI as SPI

import Adafruit_MCP3008

step 1.3: write the code for the input and output port pins that are of transmitting the signal for example: Pin 9 (VDD) -> 3V3

$$
\text { Pin } 10 \text { (VREF) -> } 3 \mathrm{~V} 3
$$

Step 1.4: code for giving an message to the admin if the battery distilled water was very low such may be of $-5 \mathrm{v}$, this were code using the "If statement "

Step 1.5: As we know that the Rasp berry pi were of existing only in CUI but as we have we to convert it into GUI unit such that the sample code were of:

def conversion(value0):

value $=$ value 0

value $=$ value $* 1960 \#$ for $5 \mathrm{~V}$

value $=$ value $/ 1000$

pic_value=value $/ 2$ \# for bargraph display

one $=$ value $/ 100$

rest_z=value $\% 100$

tenth=rest_z/10

hundredth=rest_z\%10

return one, tenth, hundredth, pic_value,value

Step 2: create a new file named and save in the directory

such as the fie name will be of: voltage.py, directory: direction: /home/pi

example : sudo nano voltage.py

step 3: enter the code in step 1 and execute in Shell command example: sudo wget http://ossyoo.com/driver/voltage.py

step 4: Run the python code using an command line as "sudo python ./voltage.py"

step 5:they will be of converting it into DC portrait by add $16.5 \mathrm{~V}$ 
Step 6:test with the different values for desired output.

After the coding the code to the Rasp berry Pi connect it to Arduino and as we know that there were used to connect it to mobile for transmitting the data through the internet[6].

While as if it is less then the $-5 \mathrm{v}$ then it will give an message to the admin twice, so that it is easy to update without even checking through it.

\section{CONCLUSION}

In this paper, the design and development of monitoring of distilled water using IOT is presented. This proposed system contains a limited source of Sensors such as voltammeter. This device were of processing and gathering the data from generator and sent the information to the admin through the internet or any WIFI server to the personal mobile numbers of the desired person in such cases they will be of knowing the volume of distilled water and add water it may require for it on time This devices were of low cost and have an accurate values as an output, more efficiency ,duration of time is low etc. they were some features of it. In future we would like to develop an automatic development of the batteries in such a way that if there were of having low volume of distilled water then automatically adding its self to their limit by storing it when the person give excess amount to it.

\section{REFERENCES}

1. Atzori, L.; Iera, A.; Morabito, G. Understanding the Internet of Things: Definition, potentials, and societal role of a fast evolving paradigm. AdHoc Netw. 2017, 56, 122-140.

2. Szumanowski and Y. Chang, "Battery management system based on battery nonlinear dynamics modeling," IEEE Trans. Veh. Technol 2008., vol. 57, no. 3, pp. 1425-1432, May 2008.

3. Irsyad N. H., Edi Leksono, M. Iqbal, FX Nugroho S., Nugraha, Deddy Kurniadi, Brian Yuliarto, Development of Battery Management System for Cell Monitoring and Protection, IEEE Int. Conf. on Elect. Engineering and Computer Science, pp. 24-25 Nov. 2014.

4. C. Hommalai and S. Khomfoi "Battery Monitoring System by Detecting Dead Battery Cells", International Journal of Science and Research, Vol.1, pp. 5-15, 2011.

5. Y. Li, L. Zhen, "Battery Management System", 2010 International Conference on Measuring Technology and Mechatronics Automation, pp. 739-741, 2010.

6. J. Cao, N. Schofield, and A. Emadi, "Battery balancing methods: A comprehensive review," in 2008 IEEE Vehicle Power and Propulsion Conference, VPPC 2008, 2008, pp. 1-6.

7. J. A. Asumadu, M. Haque, H. Vogel, and C. Willards, "Precision Battery Management System," in 2005 IEEE Instrumentation and Measurement Technology Conference Proceedings, 2005, vol. 2, no. May, pp. 1719.

8. B. Pattipati, K. Pattipati, J. P. Christopherson, S. M. Namburu, D. V. Prokhorov, and L. Q. L. Qiao, "Automotive battery management systems," 2008 Ieee Auto test con, no. September, pp. 8-11, Sep. 2008.

9. J. Chatzakis, K. Kalaitzakis, N. C. Voulgaris and S. N. Manias, "Designing a new generalized battery management system", IEEE Trans. Ind. Electron. Vol. 50, No. 5, pp. $990-999,2003$.

10. A. Sardar, H. Naseer, E. Qazi, and W. Ali "Smart Grids Wide Area Monitoring System for UPS Batteries Over
GSM" 2nd International Multidisplinary Conference For Better Pakistan Vol.1, pp. 159-158, May 2012, 2015.

11. A. S. Dhotre, S. S. Gavasane, A. R. Patil, and T. Nadu, "Automatic Battery Charging Using Battery Health Detection" International Journal of Engineering \& Technology. Innovative science vol. 1, no. 5, pp. 486490, 2014. 